\title{
Skin Lesion Segmentation in Dermoscopy Imagery
}

\author{
Shelly Garg ${ }^{1}$ and Balkrishan Jindal ${ }^{2}$ \\ ${ }^{1}$ Department of Electronics and communication, Punjabi University, India \\ ${ }^{2}$ Yadavindra College of Engineering, Computer Engineering Section, Punjabi University, India
}

\begin{abstract}
The main purpose of this study is to find an optimum method for segmentation of skin lesion images. In the present world, Skin cancer has proved to be the most deadly disease. The present research paper has developed a model which encompasses two gradations, the first being pre-processing for the reduction of unwanted artefacts like hair, illumination or many other by enhanced technique using threshold and morphological operations to attain higher accuracy and the second being segmentation by using k-mean with optimized Firefly Algorithm (FFA) technique. The online image database from the International Skin Imaging Collaboration (ISIC) archive dataset and dermatology service of Hospital Pedro Hispano (PH ${ }^{2}$ ) dataset has been used for input sample images. The parameters on which the proposed method is measured are sensitivity, specificity, dice coefficient, jacquard index, execution time, accuracy, error rate. From the results, authors have observed proposed model gives the average accuracy value of huge number of cancer images using ISIC dataset is $98.9 \%$ and using $\mathrm{PH}^{2}$ dataset is $99.1 \%$ with minimize average less error rate. It also estimates the dice coefficient value 0.993 using ISIC and 0.998 using $\mathrm{PH}^{2}$ datasets. However, the results for the rest of the parameters remain quite the same. Therefore the outcome of this model is highly reassuring.
\end{abstract}

Keywords: Automatic detection, FFA, K-mean, pre-processing, segmentation.

Received October 29, 2019; accepted February 7, 2021

https://doi.org/10.34028/iajit/19/1/4

\section{Introduction}

Skin cancer is characterize as a build up in which there is an abnormal reinforcement of certain cells that happens due to alteration in gene expression on the skin layer [34]. These cancerous cells further penetrate neighbourhood cells. In this modern world, huge number of cancer patients has been elevated because human body gets affected by various factors like life span increase, and ultraviolet light exposure or many more [38]. Skin cancer is essentially of two different types which are malignant and benign. The difference between both categories is due to their ability to spread or metastasize to remote tissues and organs. Malignant type of Cancer can effectively invade and can destroy its neighbouring tissues. It can also spread to remote tissues as well as organs through the bloodstream or lymphatic system. Most of the people are infected by this disease [30, 32]. Benign cancer is a more localized type of cancer but it can affect the environment by applying pressure on neighbouring nerves or blood vessels. The growth rate of benign cancer type is less than malignant cancer type. If these cancers are not treated properly, it may lead to harmful effects on the human body. So, skin cancer detection is the main part of proper diagnosis. For the cancer patient, a technique named as biopsy is used which is an invasive operation and gives unpleasant experience. To avoid an unnecessary biopsy, dermoscopy imaging technique is used for detailed inspection of skin layers by using a microscope and other special illumination equipment. The major problem is to determine the presence of skin lesions in dermoscopy images and to classify the skin lesion type. Few steps are required for the detection of skin lesion such as segmentation [21], feature extraction [5] and classification process [12]. In this paper, an automated segmentation technique is suggested which can be accustomed as an initial measure for skin lesion categorization. This automatic system is used to identify and to locate the skin lesion regions which are very helpful for dermatologists to detect skin cancer [14, 39]. This suggested technique comprises of the following two footsteps:

1. Pre-processing based on enhancement technique using Thresholding and morphological operations for the removal of artefacts like hair, illumination defects and ink markings.

2. Segmentation of pre-processed image using Kmean clustering with optimized Firefly Algorithm (FFA) [17]. International Skin Imaging Collaboration (ISIC) and $\mathrm{PH}^{2}$ dataset are employed for the performance analysis of this proposed technique and also evaluate some parameters such as specificity, sensitivity, dice coefficient, Jacquard index, accuracy, execution time, error [23, 37]. This paper has been methodically classified in various sections like section 2 describes the literature review. Section 3 mentions the general outline structure and describes the proposed method for segmentation of skin lesion. The section 4 describes the 
outcomes of suggested techniques and correlative study. All results and discussion are described in section 5. In the last section conclusion of this suggested work has been mentioned.

\section{Literature Review}

It has been shown by an expert specialist that the accuracy rate calculated is $60 \%$ using a visual examination method. So, because of this low accuracy rate, automatic detection system is required for skin cancer detection to achieve high accuracy rate. The basic three methods for automatic detection system are segmentation process, feature extraction process, and classification process. From these, segmentation is the main task; therefore, the accuracy in segmentation directly affects other tasks. However, skin lesion segmentation becomes extremely difficult because of variations in size, boundaries, and color of skin lesion with the distinctive sort of skin. It's very difficult to discriminate the normal skin and skin lesion. And also, Segmentation becomes more challenging because of various artefacts such as hair, remarks and so on in dermoscopy images. There are various types of segmentation and classification methods that can be resorted to resolve these kinds of issues.

Celebi et al. [10] introduced region-based, threshold-based and edge-based techniques for the segmentation and detection of skin lesion.

Ahn et al. [4] described a saliency-based lesion segmentation method for the segmentation of lesion from the skin images produced by dermoscopy equipment. This proposed technique separates the background part from skin lesion image based on color properties, boundaries, and image regions.

Sadri et al. [36] suggested a technique for skin lesion segmentation which is called a Fixed Grid Wavelet Network (FGWN). This method uses the dermoscopy image that gives RGB values which are used as an input and with that it uses orthogonal least square algorithm for the estimation of network weights. This emanates the boundary of skin lesion region.

Kechichian et al. [26] mentioned a Graph cut method for segmentation, that takes under the consideration of texture, color, and shape. With the help of the Graph cut method, the classifier is trained by driving the seed pixel from an initial segmentation of skin lesion based on Morphological and logical operators.

Abbas et al. [1] described a region-based active contour method to derive the skin lesion region.

Pennisi et al. [35] suggested the Delaunay Triangulation (DT) method for the segmentation process. This proposed method was applied to $\mathrm{PH}^{2}$ dataset.

Ma and Tavares [27] proposed a deformable model in to portion the skin lesion from the dermoscopy image. It was used to differentiate the skin lesion and regular healthy skin based on segment lesion region.

Abbas et al. [3] described the least-square and dynamic programming method to ascertain the ideal boundary of skin lesion.

Silveira et al. [40] introduced six different methods for segmentation was suggested on edgebased, threshold-based and region-based algorithms and tested on hundred melanocytic skin lesion images.

Further, Bi et al. [6] supported a segmentation technique to grounded on image-wise administered knowledge and multi-scale super pixel supported Cellular Automata (CA).

Garnavi et al. [18] suggested a skin lesion border detection arrangement which is grounded on histogram thresholding and colour space analysis. The lesion border was detected by estimation of optimal color channel with hybrid thresholding and morphological operators.

Gomez et al. [20] projected an independent histogram method to enhance the structure in skin lesion image. Skin lesion is extracted from this enhanced skin lesion image by histogram Thresholding method.

Yuksel and Borlu [45] proposed a Type-2 fuzzy logic method by which was employed to skin segment lesion image.

Fan et al. [16] introduced a technique based on the saliency map method with Ost'u threshold to extract the skin lesion border.

Zhou et al. [46] suggested a new mean shift methodology supported fuzzy c-means principle. This method was used to find out the reliable cluster centre point by calculating objective function based on the mean-field and the fuzzy c-means algorithms.

Suer et al. [41] employed an segmentation algorithm to get the better precision and used to remove unwanted data for fast computation.

Xie and Bovik [43] proposed a segmentation algorithm to obtain optimized and stabilized clustering based on genetic algorithm.

Celebi and Zornberg [9] mentioned a machine learning method to discriminate significant colours in skin images.

Celebi et al. [11] established a fast and exclusive technique is employed for detecting lesion border on the statistical region merging method .

Abbas et al. [2] had explained various hair eliminating processes alike in painting by PDE nonlinear diffusion, linear interpolation and soon.

\section{The Proposed Methodology}

An automatic learning technique is employed for skin lesion segmentation. This technique consists with two steps such as pre-processing and segmentation. The 
first step i.e., pre-processing reduces the artefacts and then this enhanced filtered image is employed for segmentation. This segmentation is done by K-mean clustering with optimized FFA algorithm. The block diagram below throws light on the workflow of the proposed method in Figure 1.

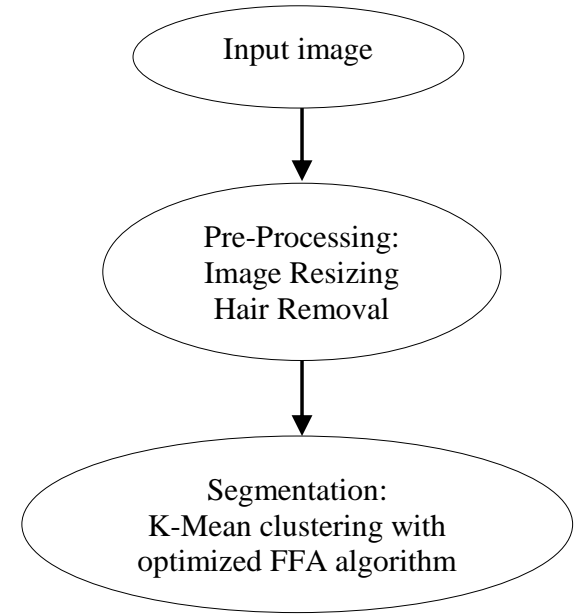

Figure 1. Framework of the proposed segmentation technique.

\subsection{Pre Processing}

It is very difficult to analyze the noisy dermoscopy images. To augment the improvement in quality of skin lesion images, Pre Processing step is used to remove the unwanted noise and eliminate irrelevant information described by [42]. Artefacts such as hairs, markers make the process of segmentation arduous. So, to enhance the preciseness of the segmentation process there is a requirement of some pre-processing steps.

\subsubsection{Image Resizing}

High resolution dermoscopy images consist with high pixel range. So, their computational complexity becomes very difficult. Therefore to minimize this computation, input high pixel images are scaled down to $50 \%$ approximately.

\subsubsection{Hair Removal}

Segmentation of lesion images becomes very difficult due to the presence of hairs because important features like texture and boundary are affected by hair pixel. Feature extraction process also becomes more difficult due to the hairs. Different types of hair like light and dark colour hairs are segmented by adaptive canny edge detector and rarefaction by morphological operator [42]. The following steps for hair removal are firstly segmentation of hair like artefacts are to be done $[19,28]$. After that proper refinement of segmented lines occurs [2]. In this paper, an enhanced technique has been applied to remove this type of artefact by using threshold [13] and morphological operations
Results of pre-processed images from the samples of ISIC are displayed in Figure 2-a) and 2-b).
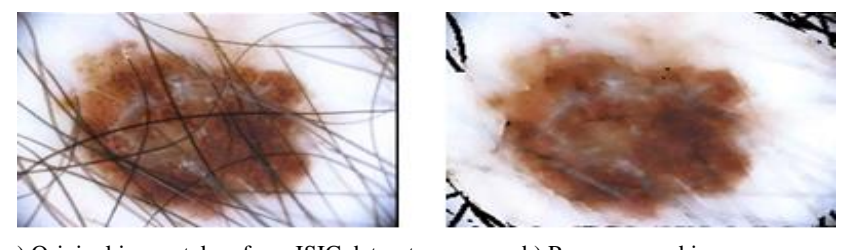

a) Original image taken from ISIC dataset.

b) Pre-processed image

Figure 2. Original and Pre-processed image.

\subsection{Segmentations}

The second step being, Segmentation is the process to detect the skin lesion border from the dermoscopy image is introduced in $[11,24]$. This part of the skin lesion contains important information of the dermoscopy image. It becomes very necessary to examine the precise defined region of lesion for diagnosis. This part differentiates the skin cancer types. Then this part is further used for feature extraction. Many techniques are used for segmentation which are edge-based, region-based and boundarybased methods [7, 8]. This suggested skin lesion segmentation comprises of two phases which are Kmean clustering to localize the exact lesion region and then this clustering gets optimized by FFA algorithm to achieve high accuracy.

\subsubsection{K-Mean Clustering}

This method is employed to cluster the pixels to obtain foreground and background region in Red Green Blue (RGB) color space [25]. This method automatically choose foremost cluster midpoint from the image pixels. Now, next cluster midpoint is selected from the remaining part of the image input pixels build on the possibility in comparison to its square stretch from the image pixels nearest to the cluster centre. The image pixels consists of interior of the lesion, background of skin lesion, background image and lesion boundary. In this method, the pixel color of cluster which is same to the skin lesion color is maintained form the obtained clusters. This method automatically pursues the information about pixel for identification of cluster as a skin lesion region. The results of $\mathrm{K}$ mean algorithm after applying on pre-processed images shown in Figure 3.

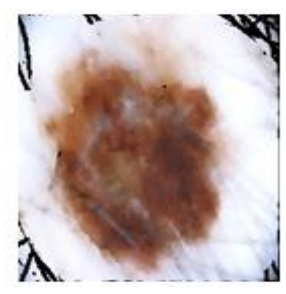

a) pre-processed image.

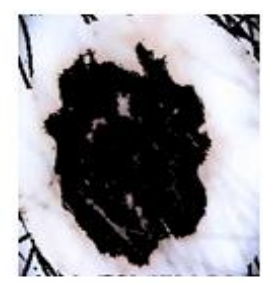

b) background region after segmentation.

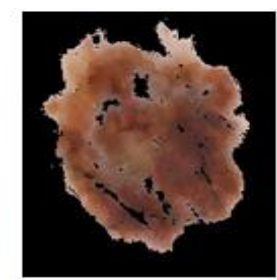

c) foreground region after segmentation.
Figure 3. Outcomes using K-Mean clustering segmentation for input image taken from ISIC dataset. 


\subsubsection{K-Mean with Optimized Fire Fly Algorithm}

FFA is the latest swarm intelligence method of this new generation [33]. It is inspired by nature, stochastic and meta-heuristic algorithm which can be used to solve the hardest optimization problem. This algorithm draws its inspiration from the community behaviour of fireflies and based on their attraction and flashing characteristics. In this paper, K-mean clustering gets optimized with an FFA algorithm to achieve the best solution. This proposed method was implemented using a Graphical User Interface (GUI) in Matlab 2014 or higher version. Pre-processing of input sample images is done by the proposed enhanced technique based on threshold and morphological operations. The threshold value of 5 is used and structural element disk is used in morphological operation to reduce artefacts. Then the K-mean clustering technique is applied to the improved image. High accuracy will be achieved with the optimization of FFA technique.

Algorithm (1) contains the functional steps of Firefly algorithm with K-Mean algorithm. The segmented image from the K-Mean algorithm is further processed by using firefly algorithm [17]. It is used to find the best solution in less population. In hybridization of K-mean and FFA, firstly K-mean method is used for segmentation and then FFA gets optimized with the results of $\mathrm{K}$-means as an input of FFA. Here FFA decides a better threshold value which can replace the wrong segment data in the output of Kmeans by selected threshold value. Figure 4-a-e) represents the steps for the segmentation of the skin lesion region using the proposed method by involving the pre-processing step and the extraction of foreground and background region using K-Mean and K-Mean with FFA technique.

Algorithm 1: Hybrid Firefly algorithm

Step 1. To Define Objective function:

$$
f(x), x=\left(x_{1}, x_{2}, \ldots, x_{d}\right) ;
$$

$f(x)$ represents fitness value function;

$x$ is no. of fireflies; $d$ defined different number of images

Step 2. For the Generation of initial population of fireflies

$$
\mathrm{x}_{\mathrm{i}}(\mathrm{i}=1,2, \ldots, \mathrm{n})
$$

here $n$ is the no. of iterations

Step 3. Light intensity $I$ is to be formulated so that it is associated with $f(x)$

Step 4. Define absorption coefficient $\gamma$

While ( $t<$ Max generation)

max generation means: maximum number of items

For $\mathrm{i}=1: \mathrm{n}$ (allfireflies)

For $\mathrm{j}=1: \mathrm{i}($ nfireflies)

I- Brightness (unique attraction)

if $\left(\mathrm{I}_{\mathrm{j}}>\mathrm{I}_{\mathrm{i}}\right)$, to find the most effective solution and

update intensity value;

$$
\text { End if }
$$

End for $j$

End for $i$

\section{End while}

End

Post- processing the results;
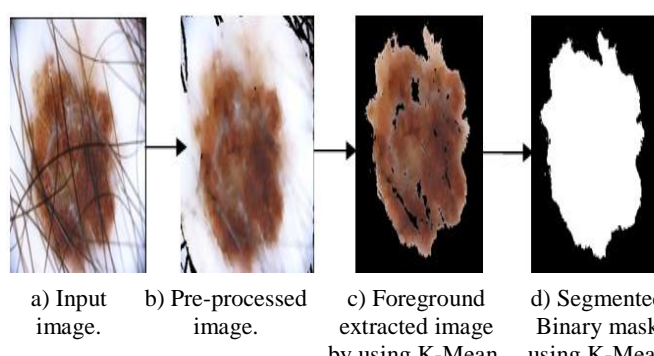

d) Segmented e) Segmented Binary mask lesion region using K-Mean using proposed with FFA. technique.

Figure 4. Various images obtained in different steps of segmentation of skin lesion region using the proposed technique.

\section{Evaluation Metrics}

Comparison of segmentation results are shown with some parameters [38] using Specificity $\left(\mathrm{S}_{\mathrm{p}}\right)$, Sensitivity (S), Dice Coefficient (DC), Jacquard Index (JI), Execution Time $\left(\mathrm{E}_{\mathrm{T}}\right)$, Accuracy $(\mathrm{ACC})[22,31]$ and Error $\left(\mathrm{E}_{\mathrm{r}}\right)$. They are defined as:

$$
\begin{gathered}
S_{p}=\frac{T_{N}}{T_{N}+F_{P}} \\
S=\frac{T_{P}}{T_{P}+F_{N}} \\
D C=\frac{2\left|G_{T} \cap A_{p}\right|}{\left|G_{T}\right|+\left|A_{P}\right|} \\
J I=\frac{\left|G_{T} \cap A_{P}\right|}{\left|G_{T} \cup A_{P}\right|} \\
A C C=\frac{T_{P}+T_{N}}{T_{N}+F_{P}+F_{N}+T_{P}} \\
\text { Er }=\frac{F_{P}+F_{N}}{T_{N}+F_{P}+F_{N}+T_{P}}
\end{gathered}
$$

Where, $T_{P}$ is that the true positive pixels represent as lesions, $G_{T}$ define ground truth, $T_{N}$ is that the true negative pixels represent as background, $A_{P}$ is that the algorithm predicted segmentation result, $F p$ is that the false positive pixels, $F_{N}$ is that the false negative pixels

\section{Results and Discussion}

In this part, the proposed method is assessed using two data sets International Skin Imaging Collaboration (ISIC) dataset consists of more than 1000 images and Hospital Pedro Hispano (PH2) dataset consists of 200 images. The act of the proposed method is measured in terms of sensitivity, specificity, dice coefficient, jacquard index, execution time, accuracy, error etc., Table 1 represents the average value of Specificity and Sensitivity of the proposed method gives better results as compare to K-Mean with Particle Swarm Optimization (PSO) $[15,29]$ and K-means [25] using ISIC data set images. These parameters are used to showcase an improvised selection of lesion region for segmentation of dataset images and are calculated 
based on the area of mask. Therefore total area takes into consideration, it would also vary but the difference between their values would remain more or less the same. It has also been observed that there is a need to focus on segmentation accuracy and error rate for producing better results. So from the analysis, it can be concluded that the fast segmentation process results in less error rate and more segmentation accuracy. Other parameters are calculated to validate the similarity of segmentation based on the dice coefficient and jacquard index technique. From the analysis, it has been found that the achieved value of the dice coefficient and jacquard Index are better in these cases which depend on the quality of skin lesion dermoscopy images [44]. The time taken by the proposed technique for the execution of results is less than K-Mean with PSO and little bit more than the K-Mean technique because of the optimized FFA algorithm. This optimized method gives high accuracy as compare to other methods. As in case of proposed method, the average error rate is very less as compare to other methods. K-Mean algorithm is complex and experimental results obtained with this algorithm are not good as results obtained with FFA. So, to reduce complexity, FFA has been used. It has been found that performance of the proposed method is quite impressive and outperformed than existing techniques. FFA is a Meta- heuristic method and is generally outperformed than simple heuristic (local search) techniques. FFA is also removed the irrelevant extract features. Also, find out the optimal solution in less population. In addition, as on the comparison with other techniques, average accuracy rate is increased by $10.9 \%$ by proposed method and average error rate is reduced by $91 \%$. Therefore, this proposed technique provides high accuracy rate with less error rate. It has high convergence rate and robust in nature. It is also used to find out the optimal solution in less population in incorporated in the paper. Table 2 represents the outcomes of the proposed technique using $\mathrm{PH}^{2}$ dataset images based on various parameters. The specificity and sensitivity of the proposed technique is better than K-Mean [25] and K-Mean with PSO technique. A high value of the dice coefficient is used for achieving better segmentation. From the analysis, it has been shown high accuracy and low error rate as compared to other methods. As in case of error rate comparison, this proposed method provides less error rate on the comparison of other methods. Figure 5-(a-f) represents the segmentation steps to locate the exact skin lesion region. Figure 5-a) input sample image Figure 5-b) Input image pre-processed to enhance the quality of the image. Figure 5-c) and Figure 5-d) Enhanced images is being segmented by K-Mean method to find out mask image and segmented image but by this method some part is missing in segmented region So, to overcome this problem. Figure 5-e) represents binary mask image using proposed method Figure 5-f) the proposed technique is used to segment the exact skin lesion region. Hence, this procedure has been applied to

various input sample images using ISIC and $\mathrm{PH}^{2}$ datasets. Figure 6-a) shows the accuracy and error rate comparison of different ISIC dataset images using KMean, K-Mean with PSO and proposed technique. From Figure 6-a) the accuracy of the proposed method is better than other techniques as well as error rate is less than other techniques. Similarly, Figure 6-b) shows the accuracy and error rate comparison of different $\mathrm{PH}^{2}$ dataset images using K-Mean, K-Mean with PSO and proposed techniques. From Figure 6-b) shows the accuracy rate of the proposed technique is better than other techniques.

Table 1. Results of pre-processed sample input images from ISIC dataset.

\begin{tabular}{|c|c|c|c|c|c|c|c|c|c|}
\hline \multirow[b]{3}{*}{ Parameters } & \multicolumn{3}{|c|}{ Results of pre-processed sample input image 1} & \multirow{2}{*}{\multicolumn{3}{|c|}{$\begin{array}{c}\text { Results of pre-processed } \\
\text { sample input image } 2 \\
\text { Techniques }\end{array}$}} & \multirow{2}{*}{\multicolumn{3}{|c|}{\begin{tabular}{|c|} 
Results of pre-processed sample input image \\
$\mathbf{3}$ \\
Techniques \\
\end{tabular}}} \\
\hline & \multicolumn{3}{|c|}{ Techniques } & & & & & & \\
\hline & $\begin{array}{l}\text { Using } \\
\text { K-mean }\end{array}$ & $\begin{array}{c}\text { K-mean with } \\
\text { PSO }\end{array}$ & $\begin{array}{l}\text { Proposed method (K- } \\
\text { Mean with FFA) }\end{array}$ & $\begin{array}{c}\text { Using } \\
\text { K-mean }\end{array}$ & \begin{tabular}{|c|} 
K-mean \\
with PSO
\end{tabular} & $\begin{array}{c}\text { Proposed method } \\
\text { (K-Mean with FFA) }\end{array}$ & $\begin{array}{c}\text { Using } \\
\text { K-mean }\end{array}$ & $\begin{array}{c}\text { K-mean } \\
\text { with PSO }\end{array}$ & $\begin{array}{l}\text { Proposed method } \\
\text { (K-Mean with FFA) }\end{array}$ \\
\hline Specificity & 0.965 & 0.972 & 0.973 & 0.964 & 0.965 & 0.966 & 0.966 & 0.969 & $\mathbf{0 . 9 7 0}$ \\
\hline Sensitivity & 0.965 & 0.980 & 0.984 & 0.969 & 0.972 & 0.979 & 0.967 & 0.970 & 0.974 \\
\hline Dice coefficient & 0.972 & 0.98 & 0.995 & 0.908 & 0.952 & 0.998 & 0.889 & 0.915 & 0.993 \\
\hline Jacquard Index & 0.901 & 0.903 & 0.907 & 0.903 & 0.904 & 0.915 & 0.903 & 0.911 & 0.919 \\
\hline Execution time & 1.033 & 88.51 & 1.112 & 0.683 & 73.64 & 1.027 & 0.707 & 91.98 & 1.032 \\
\hline Accuracy & 0.987 & 0.847 & 0.991 & 0.911 & 0.905 & 0.991 & 0.885 & 0.884 & 0.989 \\
\hline Error & 8.32 & 7.25 & 1.26 & 9.860 & 8.422 & 0.823 & 11.64 & 10.57 & 1.011 \\
\hline
\end{tabular}

Table 2 . Results of pre-processed sample input images from $\mathrm{PH}^{2}$ dataset.

\begin{tabular}{|c|c|c|c|c|c|c|c|c|c|}
\hline \multirow[b]{3}{*}{ Parameters } & \multicolumn{3}{|c|}{ Results of pre-processed sample input image 1} & \multicolumn{3}{|c|}{$\begin{array}{l}\text { Results of pre-processed } \\
\text { sample input image } 2\end{array}$} & \multicolumn{3}{|c|}{$\begin{array}{c}\text { Results of pre-processed sample input image } \\
\qquad 3\end{array}$} \\
\hline & \multicolumn{3}{|c|}{ Techniques } & \multicolumn{3}{|c|}{ Techniques } & \multicolumn{3}{|c|}{\begin{tabular}{|c|} 
Techniques \\
\end{tabular}} \\
\hline & $\begin{array}{c}\text { Using } \\
\text { K-mean }\end{array}$ & $\begin{array}{c}\text { K-mean } \\
\text { with PSO }\end{array}$ & $\begin{array}{c}\text { Proposed method } \\
\text { (K-Mean with FFA) }\end{array}$ & $\begin{array}{c}\text { Using } \\
\text { K-mean }\end{array}$ & $\begin{array}{c}\text { K-mean } \\
\text { with PSO }\end{array}$ & $\begin{array}{c}\text { Proposed method } \\
\text { (K-Mean with FFA) }\end{array}$ & $\begin{array}{c}\text { Using } \\
\text { K-mean }\end{array}$ & $\begin{array}{l}\text { K-mean } \\
\text { with PSO }\end{array}$ & $\begin{array}{l}\text { Proposed method } \\
\text { (K-Mean with FFA) }\end{array}$ \\
\hline Specificity & 0.970 & 0.973 & \begin{tabular}{|c|}
0.975 \\
\end{tabular} & 0.968 & 0.971 & $\mathbf{0 . 9 7 4}$ & 0.967 & 0.972 & 0.977 \\
\hline Sensitivity & 0.971 & 0.976 & 0.978 & 0.968 & 0.976 & 0.978 & 0.971 & 0.976 & 0.978 \\
\hline Dice coefficient & 0.930 & 0.941 & 0.993 & 0.892 & 0.905 & 0.990 & 0.875 & 0.940 & 0.993 \\
\hline Jacquard Index & 0.912 & 0.927 & 0.940 & 0.914 & 0.933 & 0.943 & 0.919 & 0.923 & $\mathbf{0 . 9 3 1}$ \\
\hline Execution time & 0.677 & 75.24 & 1.00 & 0.918 & 91.89 & 1.05 & 0.716 & 74.72 & 1.149 \\
\hline Accuracy & 0.875 & 0.880 & 0.989 & 0.910 & 0.905 & 0.991 & 0.874 & 0.872 & 0.989 \\
\hline Error & 12.49 & 11.98 & 1.075 & 8.933 & 8.476 & 0.808 & 12.74 & 10.5 & 1.09 \\
\hline
\end{tabular}




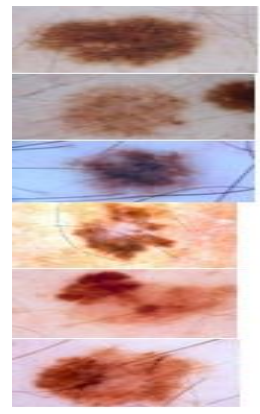

a) Input image samples.

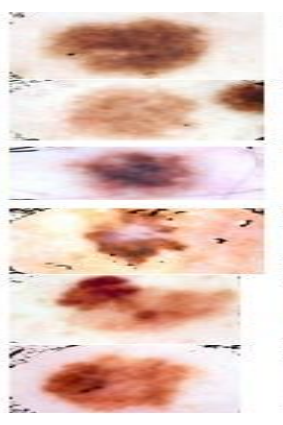

b) Pre-processed images.

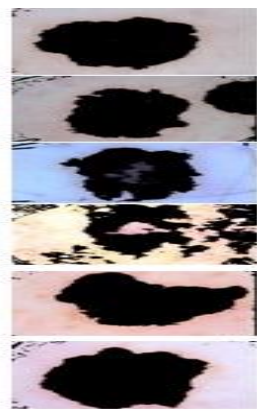

c) Mask image using K-

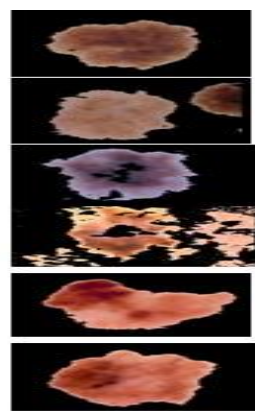

d) Segmented images using K-mean.

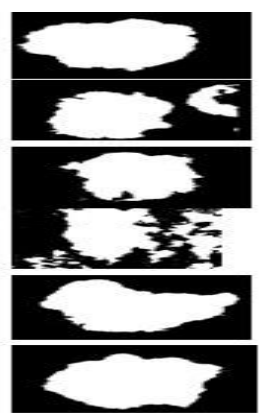

e) Binary mask images using proposed method

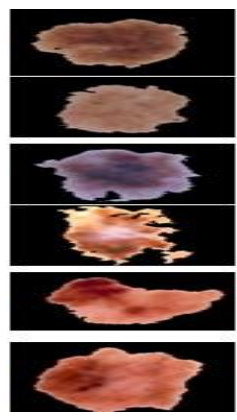

f) Segmented images using proposed method.

Figure 5. Segmentation results.

From Figure 6-a) and 6-b) it has been concluded that the proposed method is better for segmentation of skin lesion region as compared to other techniques.

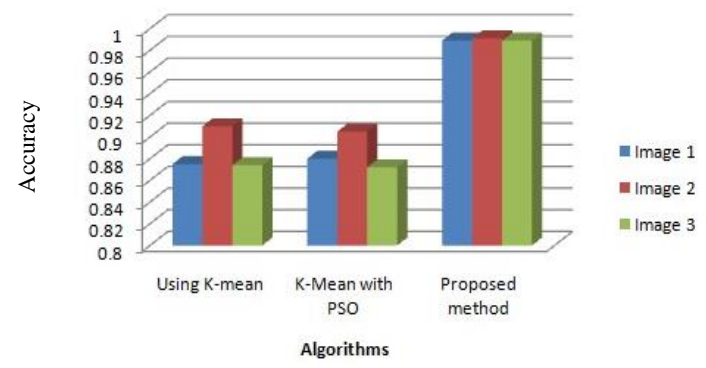

a) Accuracy comparison of different ISIC dataset images.

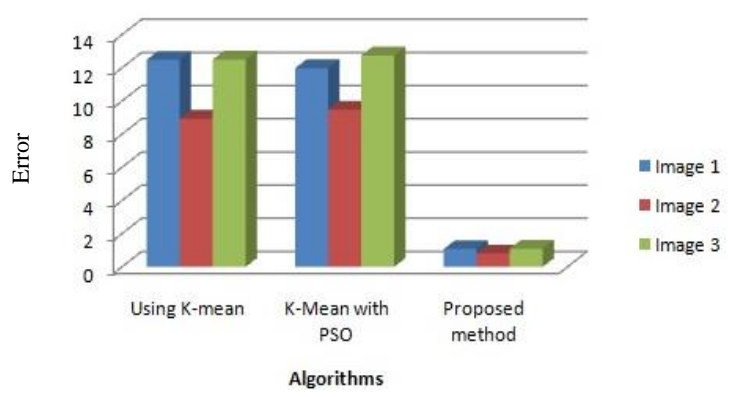

b) Error comparison of different ISIC datasets.

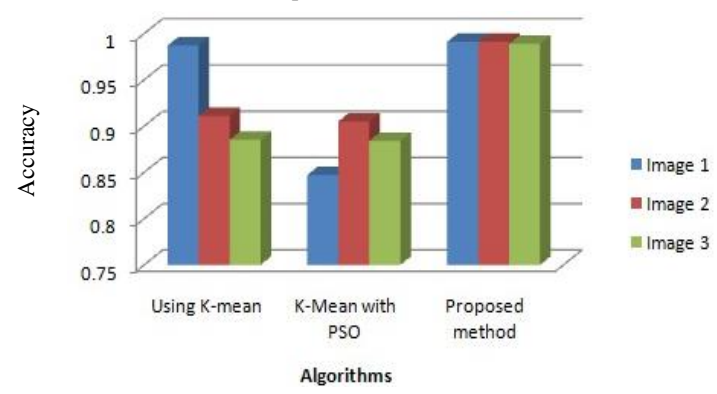

c) Accuracy comparison of different $\mathrm{PH}^{2}$ dataset images.

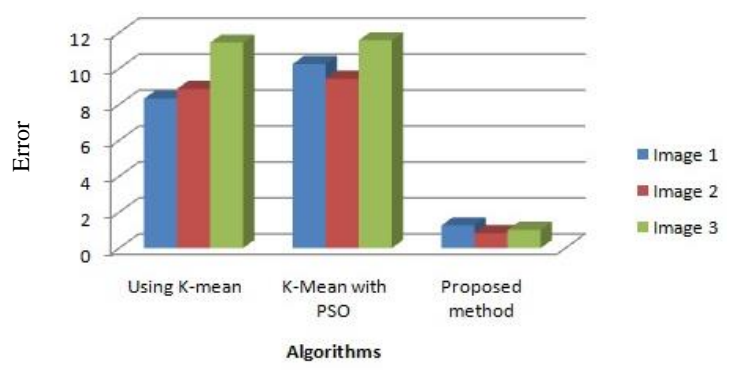

d) Error comparison of different $\mathrm{PH}^{2}$ datasets.

Figure 6. Accuracy and error comparison using different dataset images.

\section{Conclusions}

In this study, an automatic learning technique is described to segment skin lesion region. This system is useful for dermatologists to automatically find out skin lesion regions in dermoscopy images. The proposed technique consists of two steps pre-processing and segmentation. During the pre-processing, artefacts like hairs, makers are reduced by enhanced technique based on threshold and morphological operations. This preprocessed image is employed for good segmentation of skin lesion region. From the pre-processed image, the lesion region is segmented using proposed algorithm is employed to get the lesion region with improved boundaries. The proposed method is evaluated using ISIC and $\mathrm{PH}^{2}$ dataset images. From both the datasets, a good accuracy of 0.989 and 0.991 respectively has been obtained. The dice coefficient values of 0.993 and 0.998 are calculated for the ISIC dataset and $\mathrm{PH}^{2}$ dataset respectively. Therefore, it has been concluded that proposed algorithm provides higher accuracy, induces low error rate as compared to other techniques.

\section{References}

[1] Abbas Q., Fondón I., and Rashid M., "Unsupervised Skin Lesions Border Detection via Two-Dimensional Image Analysis," Computer Methods and Programs in Biomedicine, vol. 104, no. 3, pp. e1-e15, 2011.

[2] Abbas Q., Celebi M., and García I., "Hair Removal Methods: A Comparative Study for Dermoscopy Images," Biomedical Signal Processing and Control, vol. 6, no. 4, pp. 395404, 2011.

[3] Abbas Q., Celebi M., García I., and Rashid M., "Lesion Border Detection in Dermoscopy Images Using Dynamic Programming," Skin Research and Technology, vol. 17, no. 1, pp. 91-100, 2011.

[4] Ahn E., Kim J., Bi L., Kumar A., Li C., Fulham M., and Feng D., "Saliency-Based Lesion Segmentation Via Background Detection in Dermoscopic Images," IEEE Journal of Biomedical and Health Informatics, vol. 21, no. 6, pp. 1685-1693, 2017. 
[5] Barata C., Celebi M., and Marques J., "A Survey of Feature Extraction in Dermoscopy Image Analysis of Skin Cancer," IEEE Journal of Biomedical and Health Informatics, vol. 23, no. 3, pp. 1096-1109, 2018.

[6] Bi L., Kim J., Ahn E., Kumar A., Fulham M., and Feng D., "Dermoscopic Image Segmentation via Multistage Fully Convolutional Networks," IEEE Transactions on Biomedical Engineering, vol. 64, no. 9, pp. 2065-2074, 2017.

[7] Bi L., Kim J., Ahn E., Feng D., and Fulham M., "Automated Skin Lesion Segmentation Via Image-Wise Supervised Learning and MultiScale Superpixel Based Cellular Automata," in Proceedings of $13^{\text {th }}$ International Symposium on Biomedical Imaging, Prague, pp. 1059-1062, 2016.

[8] Bozorgtabar B., Sedai S., Roy P., and Garnavi R., "Skin Lesion Segmentation Using Deep Convolution Networks Guided by Local Unsupervised Learning," IBM Journal of Research and Development, vol. 61, no. 4-5, pp. 1-6, 2017.

[9] Celebi M. and Zornberg A., "Automated Quantification of Clinically Significant Colors in Dermoscopy Images and its Application to Skin Lesion Classification," IEEE Systems Journal, vol. 8, no. 3, pp. 980-984, 2014.

[10] Celebi M., Iyatomi H., Schaefer G., and Stoecker W., "Lesion Border Detection in Dermoscopy Images," Computerized Medical Imaging and Graphics, vol. 33, no. 2, pp. 148-153, 2009.

[11] Celebi M., Kingravi H., Iyatomi H., Aslandogan Y., Stoecker W., Moss R., Malters J., Grichnik J., Marghoob A., Rabinovitz H., and Menzies S., "Border Detection in Dermoscopy Images Using Statistical Region Merging," Skin Research and Technology, vol. 14, no. 3, pp. 347-353, 2008.

[12] Celebi M., Codella N., and Halpern A., "Dermoscopy Image Analysis: Overview and Future Directions," IEEE Journal of Biomedical and Health Informatics, vol. 23, no. 2, pp. 474478, 2019.

[13] Celebi M., Wen Q., Hwang S., Iyatomi H., and Schaefer G., "Lesion Border Detection in Dermoscopy Images Using Ensembles of Thresholding Methods," Skin Research and Technology, vol. 19, no. 1, pp. e252-e258, 2013.

[14] Codella N., Gutman D., Celebi M., Helba B., Marchetti M., Dusza S., Kalloo A., Liopyris K., Mishra N., Kittler H., and Halpern A., "Skin Lesion Analysis Toward Melanoma Detection," in Proceedings of $15^{\text {th }}$ International Symposium on Biomedical Imaging, Washington, pp. 168172, 2018.

[15] Eltayef K., Li Y., and Liu X., "Lesion Segmentation in Dermoscopy Images Using Particle Swarm Optimization and Markov
Random Field," in Proceedings of IEEE $30^{\text {th }}$ International Symposium on Computer-Based Medical Systems, Thessaloniki, pp. 739-744, 2017.

[16] Fan H., Xie F., Li Y., Jiang Z., and Liu J., "Automatic Segmentation of Dermoscopy Images using Saliency Combined with Otsu Threshold," Computers in Biology and Medicine, vol. 85, pp. 75-85, 2017.

[17] Francisco R., Costa M., and Rocha A., "Experiments with Firefly Algorithm," in Proceedings of International Conference on Computational Science and its Applications, Russia, pp. 227-236, 2014.

[18] Garnavi R., Aldeen M., Celebi M., Varigos G., and Finch S., "Border Detection in Dermoscopy Images Using Hybrid Thresholding on Optimized Color Channels," Computerized Medical Imaging and Graphics, vol. 35, no. 2, pp. 105-115, 2011.

[19] George Y., Aldeen M., and Garnavi R., "Skin Hair Removal for 2D Psoriasis Images," in Proceedings of International Conference on Digital Image Computing: Techniques and Applications, Adelaide, pp. 1-8, 2015.

[20] Gomez D., Butakoff C., Ersboll B., and Stoecker W., "Independent Histogram Pursuit for Segmentation of Skin Lesions," IEEE Transactions on Biomedical Engineering, vol. 55, no. 1, pp. 157-161, 2007.

[21] Goyal M., Oakley A., Bansal P., Dancey D., and Yap M., "Skin Lesion Segmentation in Dermoscopic Images with Ensemble Deep Learning Methods," IEEE Access, vol. 8, pp. 4171-4181, 2019.

[22] Grin C., "Accuracy in the Clinical Diagnosis of Malignant Melanoma," Archives of Dermatology, vol. 126, no. 6, pp. 763-766, 1990.

[23] Iyatomi H., Oka H., Saito M., Miyake A., Kimoto M., Yamagami J., Kobayashi S., Tanikawa A., Hagiwara M., Ogawa K., Argenziano G., Soyer H., and Tanaka M., "Quantitative Assessment of Tumour Extraction from Dermoscopy Images and Evaluation of Computer-Based Extraction Methods for an Automatic Melanoma Diagnostic System," Melanoma Research, vol. 16, no. 2, pp. 183-190, 2006.

[24] Jafari M., Karimi N., Nasr-Esfahani E., Samavi S., Soroushmehr S., Ward K., and Najarian K., "Skin Lesion Segmentation in Clinical Images Using Deep Learning," in Proceedings of $23^{\text {rd }}$ International Conference on Pattern Recognition, Cancun, pp. 337-342, 2016.

[25] Jaisakthi S., Mirunalini P., and Aravindan C., "Automated Skin Lesion Segmentation of Dermoscopic Images Using Grabcut and KMeans Algorithms," IET Computer Vision, vol. 12, no. 8, pp. 1088-1095, 2018. 
[26] Kechichian R., Gong H., Revenu M., Lezoray O., and Desvignes M., "New Data Model for GraphCut Segmentation: Application to Automatic Melanoma Delineation," in Proceedings of IEEE International Conference on Image Processing, Paris, pp. 892-896, 2014.

[27] Ma Z. and Tavares J., "A Novel Approach to Segment Skin Lesions in Dermoscopic Images Based on a Deformable Model," IEEE Journal of Biomedical and Health Informatics, vol. 20, no. 2, pp. 615-623, 2015.

[28] Maglogiannis I. and Delibasis K., "Hair Removal on Dermoscopy Images," in Proceedings of $37^{\text {th }}$ Annual International Conference of the IEEE Engineering in Medicine and Biology Society, Milan, pp. 2960-2963, 2015.

[29] Mekhmoukh A. and Mokrani K., "MR Brain Image Segmentation Using an Improved Kernel Fuzzy Local Information C-Means Based Wavelet, Particle Swarm Optimization (PSO) Initialization and Outlier Rejection with Level Set Method," The International Arab Journal of Information Technology, vol. 15, no. 4, pp. 683692, 2018.

[30] Melanoma Foundation (AIM), Melanoma Stats, Facts and Figures. https://www.aimatmelanoma. org/about-melanoma/melanoma-stats-facts-and figures., Last Visited, 2016.

[31] Morton C. and Mackie R., "Clinical Accuracy of the Diagnosis of Cutaneous Malignant Melanoma," British Journal of Dermatology, vol. 138, no. 2, pp. 283-287, 1998.

[32] National Cancer Institute. Cancer Stat Facts: Melanoma of the Skin. https://seer.cancer.gov/statfacts/html/melan.html, Last Visited, 2019.

[33] Nayak J., Naik B., and Behera H., "Cluster Analysis Using Firefly-Based K-means Algorithm: A Combined Approach," in Proceedings of Advances in Intelligent Systems and Computing Computational Intelligence in Data Mining, pp. 55-64, 2017.

[34] Pathan S., Prabhu K., and Siddalingaswamy P., "Techniques and Algorithms for Computer Aided Diagnosis of Pigmented Skin Lesions-A Review," Biomedical Signal Processing and Control, vol. 39, pp. 237-262, 2018.

[35] Pennisi A., Bloisi D., Nardi D., Giampetruzzi A., Mondino C., and Facchiano A., "Skin Lesion Image Segmentation using Delaunay Triangulation for Melanoma Detection," Computerized Medical Imaging and Graphics, vol. 52, pp. 89-103, 2016.

[36] Sadri A., Zekri M., Sadri S., Gheissari N., Mokhtari M., and Kolahdouzan F., "Segmentation of Dermoscopy Images Using Wavelet Networks," IEEE Transactions on
Biomedical Engineering, vol. 60, no. 4, pp. 11341141, 2012.

[37] Setiawan A., "Image Segmentation Metrics in Skin Lesion: Accuracy, Sensitivity, Specificity, Dice Coefficient, Jaccard Index, and Matthews Correlation Coefficient," in Proceedings of International Conference on Computer Engineering, Network, and Intelligent Multimedia, Surabaya, pp. 97-102, 2020.

[38] Siegel R., Miller K., and Jemal A., "Cancer Statistics, 2019," CA: a Cancer Journal for Clinicians, vol. 69, no. 1, pp. 7-34, 2019.

[39] Siegel R., Miller K., Fuchs H., Fedewa S., Butterly L., Anderson J., Cercek A., Smith R., and Jemal A., "Cancer Statisctics, 2020," CA: a Cancer Journal for Clinicians, vol. 70, no. 3, pp. 145-164, 2020.

[40] Silveira M., Nascimento J., Marques J., Marcal A., Mendonca T., Yamauchi S., Maeda J., and Rozeira J., "Comparison of Segmentation Methods for Melanoma Diagnosis in Dermoscopy Images," IEEE Journal of Selected Topics in Signal Processing, vol. 3, no. 1, pp. 3545, 2009.

[41] Suer S., Kockara S., and Mete M., “An Improved Border Detection in Dermoscopy Images for Density Based Clustering," in Proceedings of the MidSouth Computational Biology and Bioinformatics Society Conference, Texas, pp. 110, 2011.

[42] Toossi M., Pourreza H., Zare H., Sigari M., Layegh P., and Azimi A., "An Effective Hair Removal Algorithm for Dermoscopy Images," Skin Research and Technology, vol. 19, no. 3, pp. 230-235, 2013.

[43] Xie F. and Bovik A., "Automatic Segmentation of Dermoscopy Images Using Self-Generating Neural Networks Seeded by Genetic Algorithm," Pattern Recognition, vol. 46, no. 3, pp. 1012-1019, 2013.

[44] Yu L., Chen H., Dou Q., Qin J., and Heng P., "Automated Melanoma Recognition in Dermoscopy Images via Very Deep Residual Celebi, Networks," IEEE Transactions on Medical Imaging, vol. 36, no. 4, pp. 994-1004, 2016.

[45] Yuksel M. and Borlu M., "Accurate Segmentation of Dermoscopic Images by Image Thresholding Based on Type-2 Fuzzy Logic," IEEE Transactions on Fuzzy Systems, vol. 17, no. 4, pp. 976-982, 2009.

[46] Zhou H., Schaefer G., Sadka A., and Celebi M., "Anisotropic Mean Shift Based Fuzzy C-Means Segmentation of Dermoscopy Images," IEEE Journal of Selected Topics in Signal Processing, vol. 3, no. 1, pp. 26-34, 2009. 


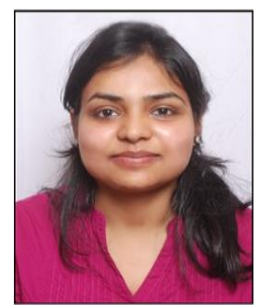

Shelly Garg received the Bachelor of Technology degree in Electronics and Communication Engineering from Punjab Technical University, MIMIT, Malout, Punjab, India in 2012, Master of Technology degree in Electronics and Communication Engineering from Punjabi University Patiala, in 2014. She is pursuing a Ph.D. at Punjabi University, Patiala, India. She is also working as an Assistant professor in SGT University, Gurgaon. She is her research interests include image processing, signal processing, microprocessors, and digital circuits. She has published various research papers in areas such as adaptive filter, biometric, adaptive algorithms, etc. She has attended various faculty development programs (FDP) and workshops for the enhancement of knowledge. She has been a vivid speaker at various at national \& international conferences. She has achieved many external and professional awards and marks of recognition. She was awarded the best paper at an international conference. She was a Fellow of The Institute of Electrical and Electronics Engineers (IEEE) and organized various activities, seminars, and conferences under IEEE

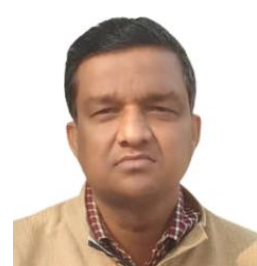

Balkrishan Jindal received the Bachelor of Technology degree in Instrumentation Engineering from Punjab Technical University, Jalandhar, Punjab, India in 1999, a Master of Technology degree in Computer Science and Engineering from Guru Jambeshbhar University Hisar, Haryana,India, in 2002, and $\mathrm{Ph}$. D. in Computer Science \& Engineering from Sant Longowal Institute of Engineering and Technology, Longowal, District: Sangrur (Punjab) India. He is working as an Assistant Professor, at Yadawindra College of Engineering, Punjabi University Guru Kashi Campus Talwandi Sabo District Bathinda, Punjab, India. He has published more than 40 research papers at various international and national level symposia/ Conferences and journals. His interests in Data hiding, Medical Imaging, and Image Processing. He is a life member of the Institute of Engineers (India), Indian Society of Technical Education (India), and International Association of Engineers (IAENG). In 2009, The Institution of Engineers (India) awarded The K F Antia Memorial Prize for his paper. 